\title{
Source-Channel Rate Allocation for Progressive Transmission of Images
}

\author{
Aria Nosratinia, Member, IEEE, Jin Lu, Member, IEEE, and Behnaam Aazhang, Fellow, IEEE
}

\begin{abstract}
Progressive image transmission is difficult in the presence of a noisy channel, mainly due to the propagation of errors during the decoding of a progressive bitstream. Excellent results for this problem are made possible through combined source-channel coding, a method that matches the channel code to the source operational rate distortion as well as channel conditions. This paper focuses on the key component of combined source-channel coding: rate allocation. We develop a parametric methodology for rate allocation in progressive source-channel coding. The key to this technique is an empirical model of decoded bit-error rate as a function of the channel code rate. We investigate several scenarios. In the case of the memoryless channel, we present closed-form expressions. For the fading channel and channels with feedback, where closed-form results are elusive, our analysis leads to low-complexity algorithms. The results presented in this paper are applicable to any progressive source code, and any family of channel codes.
\end{abstract}

Index Terms-Embedded coding, image compression, joint source-channel coding, progressive transmission, rate allocation.

\section{NOMENCLATURE}

\section{EEP Equal error protection.}

UEP Unequal error protection.

$L \quad$ Number of source bits in each packet.

$h \quad$ Packet header length.

$L^{\prime} \quad L+h$.

$r_{n} \quad$ Channel code rate for packet $n$ under UEP.

$K_{n} \quad$ Length of channel packet $n$, equal to $L^{\prime} / r_{n}$.

$P_{n} \quad$ Block error probability for packet $n$.

$r, K \quad$ Code rate and packet length under EEP.

$P_{b} \quad$ Block error probability under EEP.

$B \quad$ Overall bit budget.

$N \quad$ Total number of channel packets transmitted.

$e \quad$ Crossover probability of binary symmetric channels (BSC).

$d_{n} \quad$ Residual distortion after packet $n$.

D Distortion.

Paper approved by E. Ayanoglu, the Editor for Communication Theory and Coding Application of the IEEE Communications Society. Manuscript received December 10, 2000; revised December 21, 2001 and June 24, 2002. The work of A. Nosratinia was supported in part by the National Science Foundation under Grant CCR-9985171. This paper was presented in part at the IEEE International Conference on Image Processing, Chicago, IL, 1998, and in part at the IEEE Data Compression Conference, Snowbird, UT, 1999.

A. Nosratinia is with the Department of Electrical Engineering, University of Texas at Dallas, Richardson, TX 75080 USA (e-mail: aria@utdallas.edu).

J. Lu is with Sony Electronics Inc., San Jose, CA 95134 USA (e-mail: Jin.Lu@am.sony.com).

B. Aazhang is with the Department of Electrical and Computer Engineering,

Rice University, Houston, TX 77005 USA (e-mail: aaz@ece.rice.edu).

Digital Object Identifier 10.1109/TCOMM.2003.809256 $\alpha, \beta, \quad$ Source and channel model parameters.

$\gamma, \delta$

$X \quad$ Interarrival of successful packet receptions.

$M_{n} \quad$ Arrival process of successful packets.

$S^{(k)} \quad$ Channel state after $k-1$ state transitions.

$B^{(k)} \quad$ Number of bits transmitted during state $S^{(k)}$.

\section{INTRODUCTION}

A PROGRESSIVE bitstream can be decoded as it arrives, providing a continually improving approximation to the decoded signal. Progressive modes have been included, to various extents, in several image and video standards such as JPEG, JPEG-2000, MPEG-2, and MPEG-4. However, progressive bitstreams suffer from high sensitivity to channel errors due to error propagation at the receiver. This sensitivity arises from predictive coding and adaptive forms of variable-length coding, where the meaning of subsequent bits depend on the decoding of previous bits. Thus, a single bit error may render the remainder of the bitstream useless.

Combined source-channel coding has emerged as an effective way to address this problem. In this method, the channel code rate is carefully chosen to match the properties of source coder as well as the conditions of the channel. Sherwood and Zeger [1], [2] demonstrated a successful application of this principle to progressive image transmission. They used the set partitioning in hierarchical trees (SPIHT) compression algorithm of Said and Pearlman [3], combined with cyclic redundancy check (CRC) codes to limit error propagation and rate-compatible punctured convolutional (RCPC) codes [4] for error correction. Even though Sherwood and Zeger used pre-existing coding methodologies, their careful construction improved on previously reported results by a large margin (see [1] for list of previous works).

Rate allocation is a pivotal component of combined sourcechannel coding. To demonstrate, we use an experiment with a system similar to [1] (Fig. 1). In this experiment, we fix the channel bandwidth, thus the bit budget must be divided between the source bits and error protection. It is easy to see that achieving the right balance is critical. Too many parity bits (to the left of the peak) will leave insufficient bitrate to describe the source, while too few parity bits (to the right of the peak) will cause a catastrophic breakdown of decoding, due to uncorrected channel errors. The results reported in [1] correspond to the peak in Fig. 1, but the calculation of rate allocation (location of the peak) was left open for subsequent research.

Rate allocation for the source-channel problem has been addressed in several contexts. Qian et al. [5] proposed a gradient- 


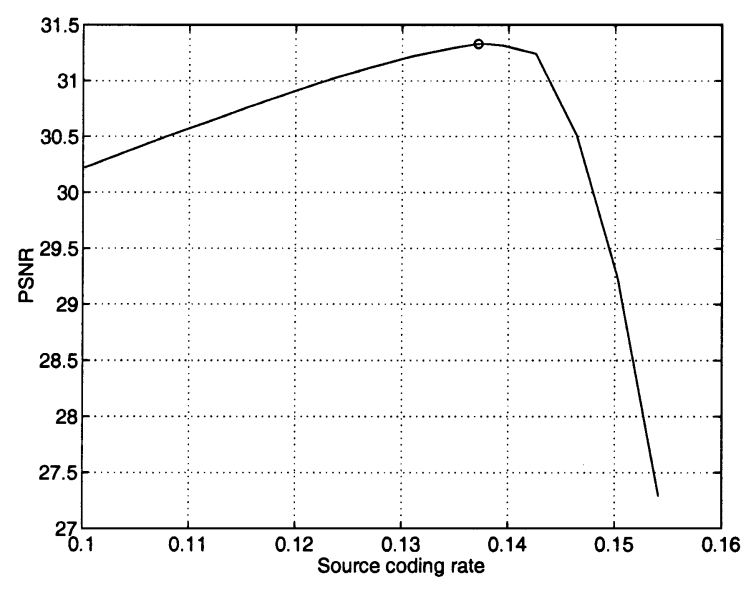

Fig. 1. Source-channel rate allocation and end-to-end peak signal-to-noise ratio (PSNR). Experiment on $512 \times 512$ Lenna.

based optimization technique for bit allocation in wireless video transmission. Chande and Farvardin [6] used dynamic programming for progressive image transmission over noisy channels. The dynamic programming approach was extended in [7] to jointly optimize block length and channel code rate.

In this paper, we develop a parametric approach for the progressive source-channel rate allocation problem. The key to this work is an accurate empirical model of decoded bit-error rate (BER) in terms of the channel code rate. This model-based approach allows simplified expressions for the relationship of the overall bitrate and distortion. Our parametric bit allocation is generally less complex than comparable nonparametric methods; thus, we believe one of the contributions of this paper is to enable the practical implementation of progressive source-channel coding. Our formulation of the problem is applicable to any progressive source code and any family of channel codes. For example, unlike [6], the use of punctured codes is not strictly required in our approach, although it is certainly possible (in fact, our examples use rate-compatible punctured codes).

We consider four different classes of BSC: channels with and without feedback, and with and without fading.

In the case of memoryless BSC without feedback, we use a Lagrangian analysis to arrive at closed-form expressions for rate allocation.

In the case of fading channels without feedback, the channel state is unknown at the encoder, and progressive transmission precludes the use of an interleaver. Under these restrictions, we consider the pragmatic approach of allocating rate according to the worst-case conditions in the channel. This may seem an unglamorous solution, but interestingly, our simulations show that this minimax approach is also near-optimal in terms of $a v$ erage distortion.

In the case of memoryless BSC with feedback, we find a rate allocation defined by the minimum of a one-dimensional (1-D) integral of a scalar variable. Although this in itself is not a closed-form solution, its calculation via 1-D optimization is much simpler than a nonparametric solution.

Finally, we consider the case of fading channels with feedback. In fading channels, automatic repeat request (ARQ) feed- back is doubly useful: not only does it enable retransmission of corrupted data, it also provides (partial) channel state information for the encoder. We show that, through an approximation, rate allocation in fading/ARQ channels can be solved via a simplified set of 1-D optimizations over fading states.

In general, the presence of feedback changes the optimal rate allocation, resulting in higher code rates for error-correcting codes and smaller overall distortion. Simulations on memoryless and fading channels show that in our framework, feedback provides up to $1-\mathrm{dB}$ improvement in PSNR.

\section{A. Preliminaries}

A typical setup for our problem is shown in Fig. 2. The progressive bitstream is characterized by its operational rate-distortion curve. This curve is available at the encoder for each transmitted image. The channel encoder is assumed to be capable of generating a family of error-correcting codes with varying rates. We do not assume that this family is rate compatible (embedded). Given the source rate-distortion curve and the statistical properties of the channel, we aim to determine the channel coding rate that will give the best end-to-end image quality, according to a distortion criterion.

In our examples throughout this paper, we use a popular wavelet image compression algorithm known as SPIHT [3], RCPC codes [4] for error correction, CRC error detection, and list Viterbi decoding [9]. Note, however, that these codes serve only as examples. The methodology of the paper is general and applicable to any progressive source, and any family of channel error-correcting codes.

As mentioned earlier, the progressive bitstream ceases to be useful past the first unrecoverable error. In fact, the decoded image quality will likely decrease after a bit error, since the decoder will interpret the post-error bitstream incorrectly. Therefore, roughly speaking, the task of a progressive transmission system over a noisy channel is two-fold: to push back the location of the first unrecoverable error as far as possible; ${ }^{1}$ and ensure that this error is discovered at the decoder, so that the decoding is stopped at that point. Rate allocation addresses the former task. To achieve the latter, the source bitstream is divided into packets, and each packet is equipped with error detection.

We consider the case of time-invariant as well as time-varying rate allocation. Time-invariant allocation has the advantage of simplicity, while time-varying rate allocation has better performance. To ensure accurate decoding in the case of time-varying rate allocation, the instantaneous rates must be transmitted to the decoder as side information. The rate required for this overhead is small; related details are discussed in Section II-B.

Strictly speaking, the motivation to push back the location of the first error applies only to channels without feedback. In a channel with feedback, error detection does not stop the decoding, but triggers a retransmission. This continues as long as the bit budget is not exhausted and the delay is within a tolerance limit. We will address the case of channels with feedback in Section III.

\footnotetext{
${ }^{1}$ To be precise, maximizing the average number of bits up to the first error produces a near-optimal (but not optimal) value for distortion. This is due to the curvature of $D(R)$.
} 


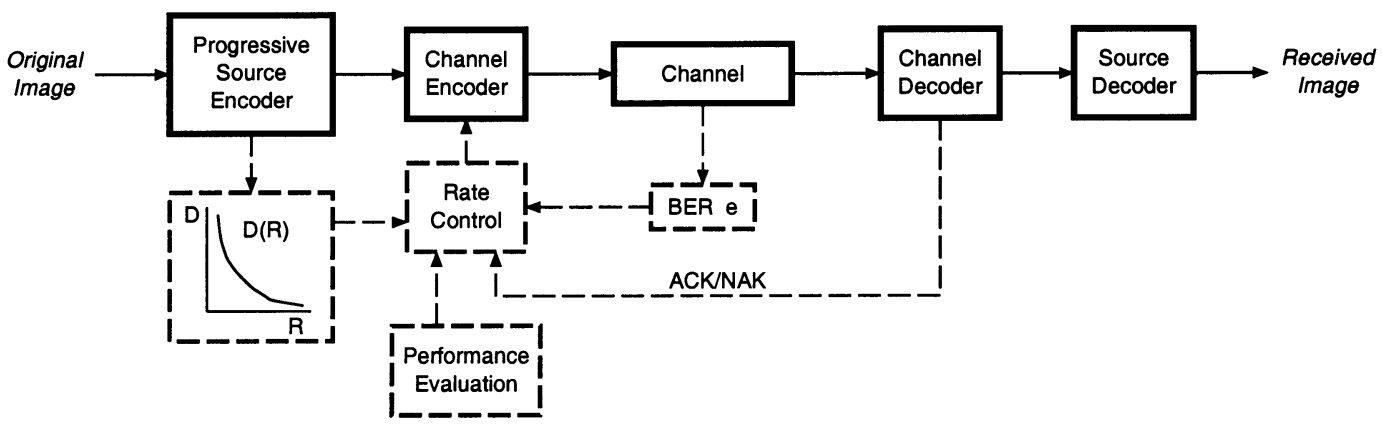

Fig. 2. Progressive source-channel coding system.

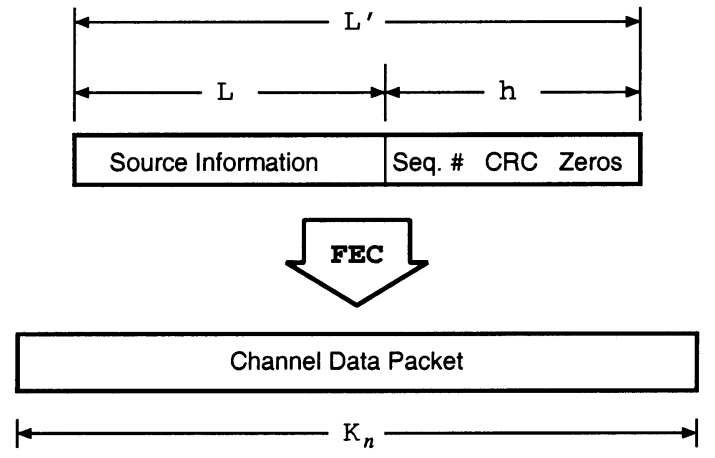

Fig. 3. Source and channel data packet structure.

\section{Channels Without FeedBack}

\section{A. Memoryless BSC}

The packetization scheme is as follows. The progressive source bitstream is divided into blocks of length $L$, and each source block is supplemented before channel coding (see Fig. 3). The bits denoted "CRC" are error-detection parity bits. Also, whenever convolutional codes are used, the encoder/decoder memory must be flushed at the end of each block, requiring a number of zeros to be inserted. The total number of bits spent for CRC and zero padding is denoted $h$. The total length of the block before error-correction coding is $L^{\prime}=L+h$.

The channel code rate for the $n$th block is denoted by $r_{n}$. The channel code maps each source block of length $L^{\prime}$ to a channel packet of length $K_{n}$, so that the $n$th channel packet length is $K_{n}=L^{\prime} / r_{n}$.

In a memoryless BSC, we denote the crossover probability (channel BER) by $e$ and the block-error rate by $P_{n}$. As is customary, $P_{n}$ is defined as the probability of at least one bit error in the $n$th block after decoding. Given that $L^{\prime}$ is fixed, the channel code rate is inversely proportional to the $K_{n}$. Thus, the blockerror rate is determined by $K_{n}$ and $e$. We assume a log-affine model for the relationship of the probability of block errors and the length of the channel packets, depending on the value of $e$, i.e.,

$$
\log P_{n}=\alpha K_{n}+\beta
$$

where $\alpha$ and $\beta$ are parameters acquired through offline simulations and stored in a lookup table at the encoder. Thus, the calculation of $\alpha, \beta$ has no bearing on the computational complexity of the encoder. Fig. 4 illustrates the match between the

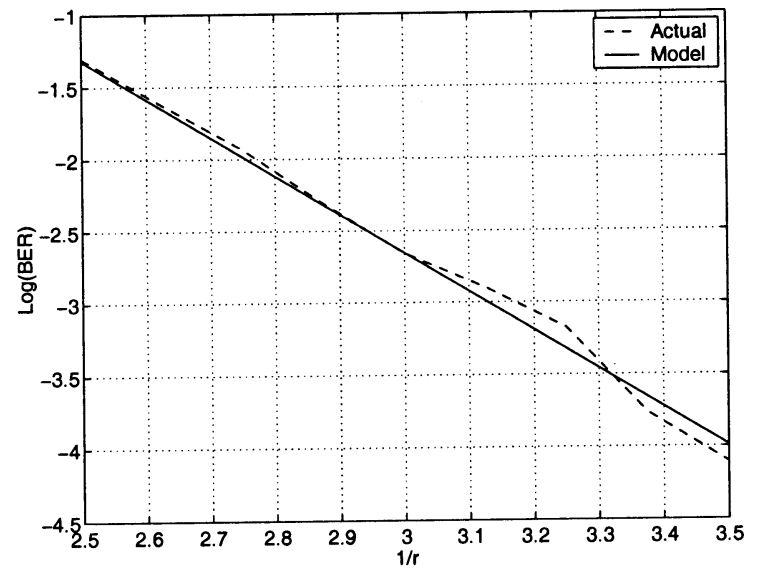

Fig. 4. Accuracy of logarithmic model for block-error rates. Error rates shown in $\log _{10}$ scale.

actual and model-based values for the RCPC codes used in our experiments.

After successfully decoding all blocks up to (and including) block $n$, the decoded signal will have a remaining distortion that is denoted by $d_{n}$. The average distortion $D$ can be written in terms of the probability of error-free decoding of exactly $n$ blocks (first decoding failure at block $n+1$ ). This probability is equal to $P_{n+1} \prod_{i=1}^{n}\left(1-P_{i}\right)$. Keeping in mind that the first and last block need special treatment, the total expected distortion for a memoryless channel is

$$
D=d_{0} P_{1}+\sum_{n=1}^{N-1} d_{n} P_{n+1} \prod_{i=1}^{n}\left(1-P_{i}\right)+d_{N} \prod_{i=1}^{N}\left(1-P_{i}\right)
$$

where $N$ is the total number of channel blocks in the transmitted stream. We need to minimize the distortion, subject to a constraint on the total bitrate, namely

$$
\begin{array}{r}
\min _{\left\{K_{n}\right\}, N} d_{0} P_{1}+\sum_{n=1}^{N-1} d_{n} P_{n+1} \prod_{i=1}^{n}\left(1-P_{i}\right)+d_{N} \prod_{i=1}^{N}\left(1-P_{i}\right) \\
\text { s.t. } \quad \sum_{n=1}^{N} K_{n}=B
\end{array}
$$

where $\left\{d_{n}\right\}$ are obtained from the operational rate-distortion curve of the source coder. These curves are easily available when the images are encoded. Assume $B$ bits are available for transmission of the image. This total bandwidth must be divided between the source coder and the channel coder, 


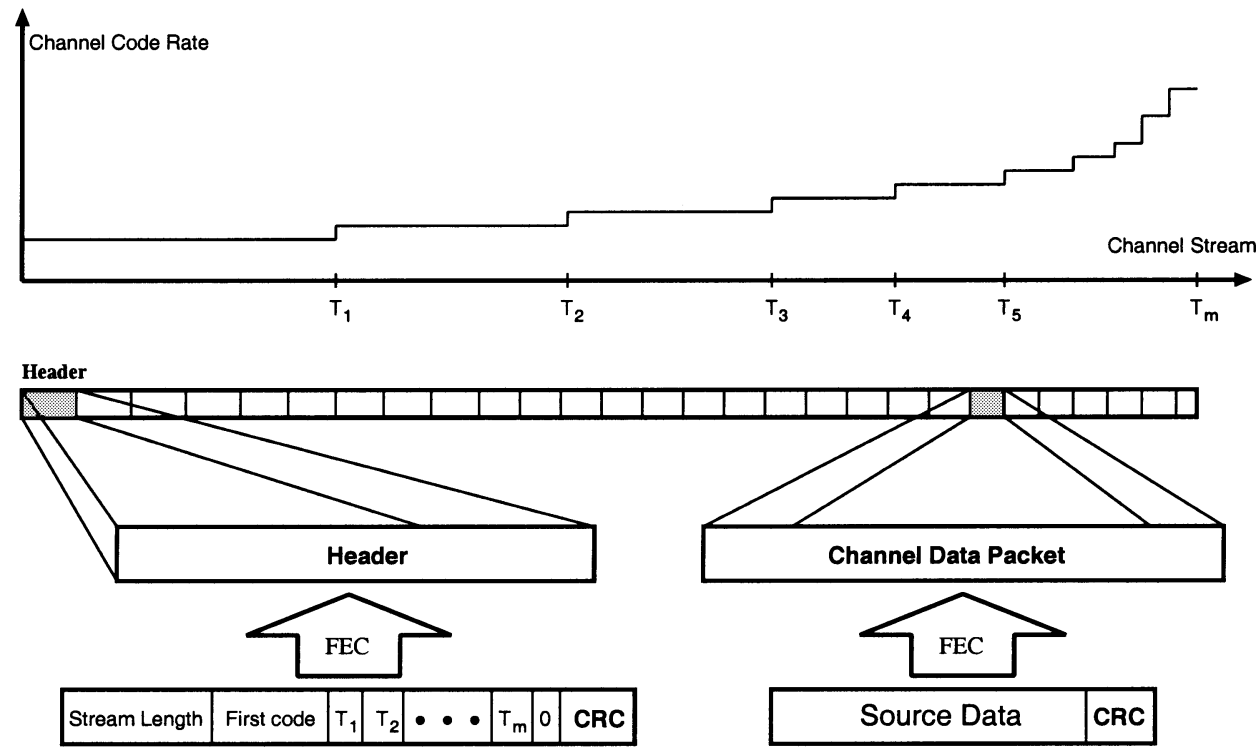

Fig. 5. Structure of the header and data packets. Header indicates the transition points in channel rate.

leading to an optimization over $\left\{K_{n}\right\}$ and $N$. We have assumed that the number of source bits per packet $L$ is fixed, therefore $N$ determines the overall tradeoff between source coding bits and channel protection bits, and $\left\{K_{n}\right\}$ determines how the total available channel protection is spread over different blocks. For fixed $N$, the solution lies at the equilibrium of the Lagrangian, which yields the recursive expression

$$
\left(\frac{1}{P_{j+1}}-\frac{1}{P_{j}}\right)(-\lambda P)=\left(d_{j}-d_{j-1}\right) \prod_{i=1}^{j}\left(1-P_{i}\right) .
$$

A proof is provided in the Appendix . The analysis presented for memoryless channels is completely general, and can be applied with any progressive source code and any family of channel codes.

To summarize, the rate allocation algorithm for the memoryless BSC is as follows:

1) Initialize $N=1, D=\infty$.

2) Initialize $K_{n}$ by allocating $B$ equally across all packets.

3) Use (4) to compute new rate allocation: update $P_{i}^{(k)}$ and get $P_{i}^{(k+1)}$, where $k$ is the iteration index, until $\sum_{i}\left(P_{i}^{(k+1)}-P_{i}^{(k)}\right)^{2}<\varepsilon$. 4) Increment $N$, go to step 2, repeat until $D$ in (2) no longer decreases.

\section{B. Rate Overhead}

In general, the solution for $K_{n}$ from the preceding analysis is not constant over $n$, i.e., different codes may be used for different packets. ${ }^{2}$ Therefore, the decoder must know the code used for each packet. This requires the transmission of some overhead information to the decoder. To limit the impact of this overhead, we take advantage of the following facts: a) there are a fi-

\footnotetext{
${ }^{2}$ It is possible to restrict the analysis to a special case of equal error protection and avoid the complication of the overhead. In that case, all preceding developments will repeat, except for replacing $K_{n}$ with $K$.
}

nite number of channel codes; $\mathrm{b}$ ) there are, at most, $N$ different rates, one per each packet; and c) the channel error protection is monotonically nonincreasing, so we only need to convey the position of rate changes in the packet stream.

The header string starts with the number of blocks, followed by the index of the first code rate, and the location of transitions to higher rates (see Fig. 5). Transition $T_{i}$ 's are represented by their distance from the end of the stream, motivated by the fact that transitions are more dense at the end of the stream. Since $T_{i} \geq T_{i-1}$, the number of bits needed to specify $T_{i}$ is no more than $\left\lceil\log _{2}\left(T_{\text {end }}-T_{i}\right)\right\rceil$. This overhead is protected strongly, with error probability better than $10^{-6}$. The header is relatively small; even though it is strongly protected, its impact on overall bit rate is negligible.

\section{Numerical Results}

In the following simulations, the system specified in Section I-A is used. The "Lenna" image is transmitted through a memoryless BSC with BER $=0.1$. We obtained block-error rate probabilities and calculated the parameters $\alpha$ and $\beta$ in (1). This enables us to solve (3) using the iterative algorithm given in Section II-A.

Fig. 6 shows the optimal error protection profile at $\mathrm{BER}=0.1$ and transmission rate 0.2 bits per pixel (bpp). Because channel codes are not available at arbitrary rates, the optimal values for the rate must be quantized. The two staircase characteristics represent rounding to the nearest rate and the nearest lower rate. For performance comparison, we also present the fixed-code rate solution (equal error protection). The final end-to-end distortion of the system is tested through Monte Carlo simulations, and presented in Table I. The impact of overhead on bit rate is approximately $0.001 \mathrm{bpp}$ (262 bits for a $512 \times 512$ image).

Our experiments indicate up to $0.3-\mathrm{dB}$ gain for a variable-rate strategy, compared to fixed rate (see Table I). Most of this gain is at lower transmission rates. Loosely speaking, the variable-rate strategy will save some bitrate while maintaining the overall 


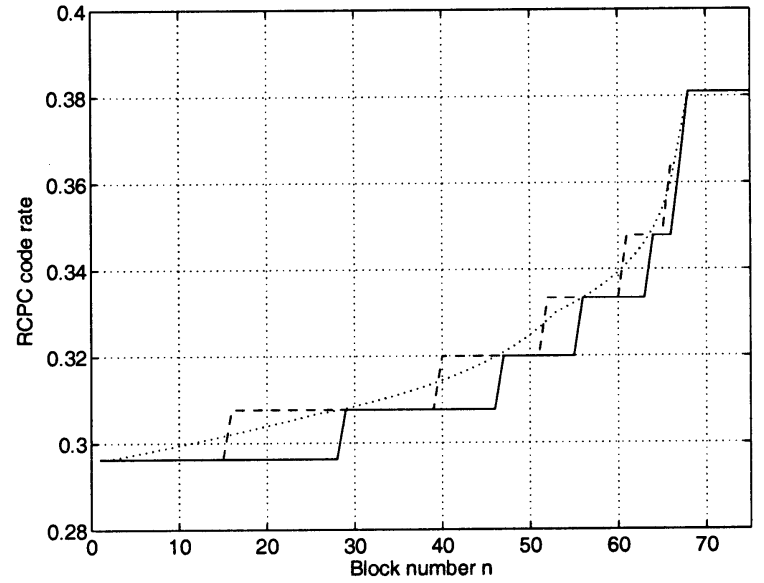

Fig. 6. Optimal code rate profile. Memoryless channel, $e=0.1$, $B=0.2 \mathrm{bpp}$. The two staircase curves represent rounding to the nearest rate and the nearest lower rate.

TABLE I

AvERAGE PSNR OVER MEMORYLESS BSC WITH BER 0.1

\begin{tabular}{|c|cccccc|}
\cline { 2 - 7 } \multicolumn{1}{c|}{} & \multicolumn{6}{c|}{ Transmission Rate } \\
\hline fixed & 0.1 & 0.2 & 0.4 & 0.6 & 0.8 & 1.0 \\
variable & 25.33 & 27.64 & 30.23 & 32.00 & 33.20 & 34.24 \\
\hline
\end{tabular}

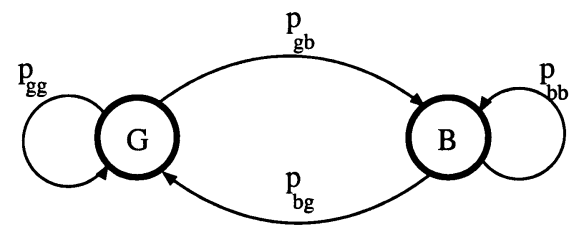

Fig. 7. Gilbert-Elliott model.

level of error protection. The saved bits are then used for a better description of the source. This effect is important primarily at lower source bitrates, where the rate-distortion curve is steep, so that additional bits make a big difference.

\section{Fading Channels Without Feedback}

In the case where no feedback is available, the encoder cannot have any information about the instantaneous state of the channel. At best, it can know about the statistics of the states of the channel. These statistics, at the bit level, are often characterized through the Gilbert-Elliott model [10]-[12] (Fig. 7).

In this model, the channel alternates between two states known as the "good" and "bad" states. It is assumed that we know the BER of the channel in each state, as well as the transition probabilities of the Markov model, but the encoder does not know the actual time-varying state of the channel. The prudent engineering choice in this situation is to design for the worst case of the channel. We demonstrate that this minimax approach is also near-optimal in the average sense for cellular wireless channels where "good" and "bad" states are very different.

Because the instantaneous variable BER is not known at the encoder, we devise a parameter called the "design BER" $\epsilon_{d}$. We
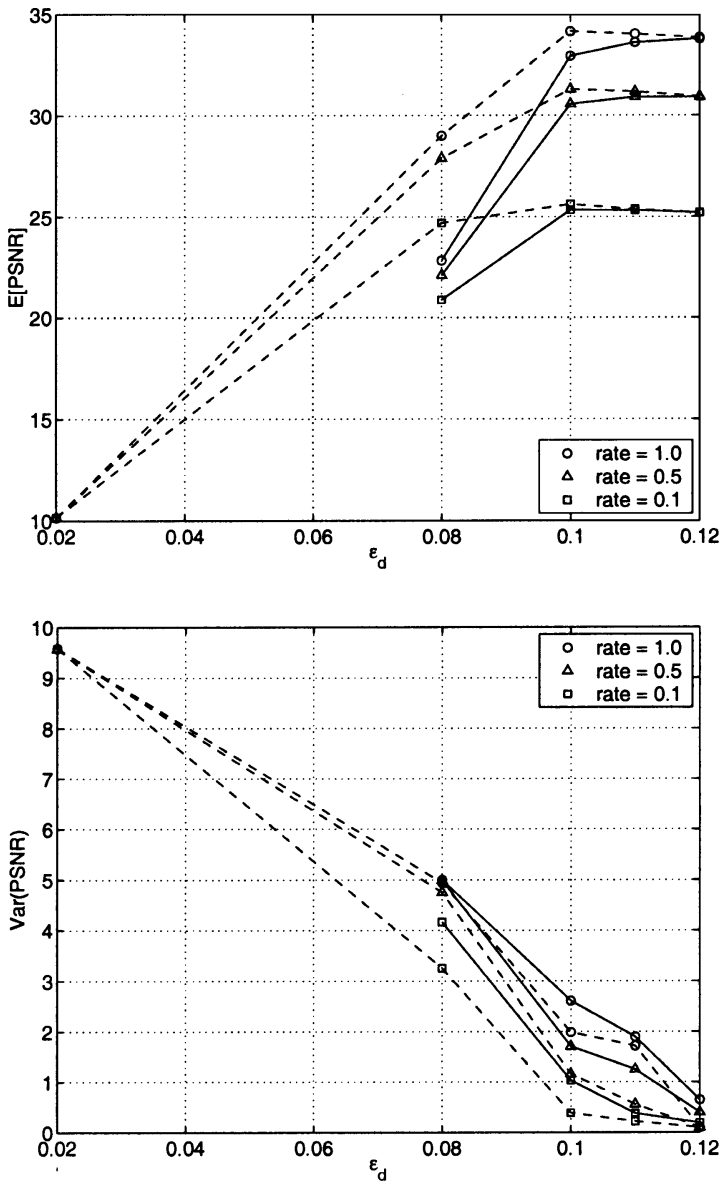

Fig. 8. Average PSNR (top) and variance of PSNR (bottom) for fading channels under different design BER. Solid line is for Channel 1, where the "bad" state dominates, dashed line for Channel 2, where the "good" state dominates.

TABLE II

BER AND MARKOV PARAMETERS FOR FADING CHANNEL

\begin{tabular}{|c|cc|cc|}
\cline { 2 - 5 } \multicolumn{1}{c|}{} & $e_{g}$ & $e_{b}$ & $P_{g b}$ & $P_{b g}$ \\
\hline Channel 1 & 0.001 & 0.12 & 0.005 & 0.0031 \\
Channel 2 & 0.001 & 0.12 & 0.00125 & 0.005 \\
\hline
\end{tabular}

find the value for this $\epsilon_{d}$ that maximizes the performance of the system. A detailed set of simulations for the fading channel are presented in [13], where a variety of cases are considered. Here, we present a summary of these simulation results in Fig. 8. The channel conditions for these experiments are given in Table II. We simulated two channels, both of which have $\mathrm{BER}=10^{-3}$ for the "good" state, and BER $=0.12$ for the "bad" state. In Channel 1, the "bad" state dominates, whereas in Channel 2, the "good" state is dominant. Fig. 8 shows that in both cases, the average distortion is optimal or near-optimal when the design BER is selected to be $\epsilon_{d}=e_{b}$. Furthermore, the standard deviation of the end-to-end PSNR, a measure of the reliability of the individual transmission, is best when $\epsilon_{d}=e_{b}$.

\section{Channels With FeEdBaCK}

The existence of feedback is helpful in two ways. First, the feedback information can be used to retransmit a subset of the erroneously received information, therefore, one can relax the 
forward error protection. This releases some portion of the bitrate, which can then be used to transmit more source symbols. Second, the feedback information can be used to estimate the state of the time-varying channel. Matching the channel code to the current channel condition provides another way to improve performance.

We saw earlier that rate allocation is critically important in channels without feedback; the same is true for channels with feedback. Prior work on the computation of rate for source and channel coding includes empirical studies, e.g., [14]-[16], as well as the more systematic works of [17]-[19]. The latter algorithms use only the channel BER, and have no need for the source parameters (aside from assuming embeddedness).

\section{A. Memoryless Channels With Feedback}

Our study of rate allocation in channels with feedback uses an extension of our technique for channels without feedback. The packetization for this case is similar to the feed-forward case. The progressive source stream is divided into blocks of length $L$, there are $h$ header bits per packet, and after error protection, the length of the channel packets is $K$, which we assume to be fixed (equal error protection for all packets). The goal is to minimize the end-to-end distortion subject to a given overall bit budget $B$. At this point, we do not consider transmission delay.

In the binary symmetric channel, we denote $e$ as the crossover probability and $r$ as the channel code rate. Therefore, $K=$ $L^{\prime} / r$. The channel block-error rate, $P_{b}$, is a function of $r$ and $e$. Similar to (1), for a given crossover probability $e$, the relation between block-error rate and inverse of code rate has a log-affine characteristic

$$
\log P_{b}=\frac{\alpha}{r}+\beta
$$

where $\alpha$ and $\beta$ are channel parameters that depend on $e$.

In a feedback scheme, some of the packets going over the channel are retransmissions due to channel errors. After $n$ packets have been sent over the channel, the number of successfully received (unique) packets will be $M_{n}$, whose distribution depends on $e$ and $r$. Because of independent channel errors, the interarrival process of two successfully received packets is independent and identically distributed (i.i.d.) with finite variance, and the number of successfully received packets $M_{n}$ is a counting process. Therefore, as $n \rightarrow \infty, M_{n}$ converges to a Gaussian random variable [20]. Asymptotically, this process has mean $\mu_{M}=n\left(1-P_{b}\right)$ and variance $\sigma_{M}^{2}=n P_{b}\left(1-P_{b}\right)$, where $P_{b}$ is the error rate of channel packets and $n$ is the number of transmitted packets, as demonstrated in the Appendix.

Now consider the case $n=N$, where $N$ is the total number of packets transmitted for one image over the channel. In the following, we use the notation $M=M_{N}$ for simplicity. We model the operational distortion-rate characteristics of the source encoder with an exponential function

$$
D(M)=10^{\gamma M+\delta}
$$

where $\gamma$ and $\delta$ are constants, and $\gamma \leq 0$. This approximation is reasonable because we generally only need to match part of the distortion-rate curve of an image. Experiments show that a very good match can be achieved for a wide variety of images.
In adopting this approximation, it is also reassuring to know that asymptotically, the distortion $^{3}$ of a wide class of sources decays exponentially with rate [21], [22].

Under this model, $D(M)$ has a log-normal distribution, i.e.,

$$
f_{D}(d)=\frac{1}{d}\left(\frac{\log e}{|\gamma|}\right) \frac{1}{\sqrt{2 \pi} \sigma} e^{-(\log d / \gamma-\delta / \gamma-\mu)^{2} / 2 \sigma^{2}}
$$

It is not difficult then to calculate the average end-to-end distortion

$$
E[D]=\frac{\log e}{|\gamma|} \int_{0}^{\infty} \frac{1}{\sqrt{2 \pi} \sigma} e^{-(\log x / \gamma-\delta / \gamma-\mu)^{2} / 2 \sigma^{2}} d x
$$

Therefore, under a given transmission rate and channel BER $e$, the optimal bit allocation problem for a BSC can be formulated as

$$
\min _{r} E[D]
$$

Note that in (8), $\mu=\mu_{M}$ and $\sigma=\sigma_{M}$ are related to $r$ through (5), (20), and (21). Problem (9) can be solved numerically to yield the optimal $r$, the rate of the channel error-correction code.

The problem (9) does not admit a closed-form solution, however, the cost function is merely a 1-D integral of a scalar variable, whose minimization is much easier than the multidimensional optimization problems arising from a nonparametric treatment of the rate allocation problem.

\section{B. Fading Channels With Feedback}

We characterize the fading channel with the Gilbert-Elliott model (Fig. 7). In each state $i$, the channel is modeled as a BSC with a fixed BER $e_{i}$. For each state $i$, the encoder can use a (potentially) different code rate $r^{(i)}$. In general, solving for $r^{(i)}$ requires an optimization whose complexity grows as $O\left(|\mathcal{C}|^{I}\right)$, where $|\mathcal{C}|$ and $I$ are, respectively, the number of distinct channel codes and distinct channel states. This optimization is prohibitively expensive.

To perform the optimization, we make an approximation. We assume that maximizing the number of successfully decoded source bits (equivalently, throughput of the ARQ system) is approximately the same as minimizing the distortion. Because the source bitstream is progressive, the more the decoded bits, the smaller the distortion

$$
\min _{r} E[D(R)] \approx D\left(\max _{r} E[R]\right) .
$$

This is equivalent to a local first-order (linear) Taylor approximation to $D(R)$.

Assuming $X_{k}$ is the interarrival process of packets (the number of transmissions until the next successful packet reception), and $\mu_{x}=E[X]$ is the average number of transmissions per successful reception, we define a new parameter, $\mu_{x} / r$, which we call the average effective transmission number. Conditioned on the state of the channel, maximizing the number of successfully received packets is equivalent to minimizing the average transmission number. This result is demonstrated in the Appendix.

\footnotetext{
${ }^{3}$ For distortion measures expressible as a power of Euclidean distance.
} 
Now it is possible to show the following decoupling result: Suppose a code rate $r^{(i)}$ is designated for each channel state $e_{i}$ in a fading channel, $i \in \mathcal{I}=\{1,2, \ldots, I\}$. Under slow fading, the I-dimensional joint optimization problem

$$
\min _{r^{(1)}, r^{(2)}, \ldots, r^{(I)}} E[D]
$$

is reduced to I 1-D problems, each corresponding to one channel state. A proof is given in the Appendix.

Our confidence in channel state estimation, and thus, the validity of decoupled optimization, is justified in the following. When a channel packet is successfully decoded, the channel state is estimated by computing the average BER within that packet. Since the typical BER of the bad state in our examples is around 0.1 or better, and the channel packet length is around 500-700, the channel state classification through BER sample mean is sufficiently accurate. Simulations show that the probability of incorrect state classification via this method is below $0.02 \%$. After classification, the computed code rate can be sent back to the transmitter via the feedback channel.

If the receiver experiences an unrecoverable error in a packet, the encoder and decoder assume a bad channel state for the next transmission, regardless of the code used in the last transmission. This strategy is reasonable, since the probability of unsuccessful reception in the good state is very small with either of the two designated codes.

\section{Feedback Scheme and Retransmission Delay}

The effects of delay depend largely on the feedback strategy. Two widely used schemes are stop-and-wait and selective repeat [8]. The stop-and-wait scheme is easy to implement, however, it has longer average delay before any one packet is successfully decoded. Selective repeat is more efficient in terms of overall delay when the transmission/propagation delay of packets is dominant and there is no strict delay jitter constraint. However, that is not true in progressive image transmissions.

In fact, in a progressive image transmission system with relatively high channel BER, the stop-and-wait scheme is more appropriate. Suppose that the encoding/decoding of one packet takes much longer than the transmission and propagation of that packet. This is a practical assumption. In the wireless channel with feedback, for example, the decoding time easily dominates the transmission time for each packet. Further assume that encoding is $n$ times faster than decoding. This is another practical assumption which is true when advanced error-protection decoding, such as list decoding or turbo decoding, is in place. At each unsuccessful reception, the decoder sends back a repeat-request signal (NAK). When selective repeat is used, the $i$ th retransmission packet arrives $m(i)$ packets after the corresponding NAK was sent, where $m(1)=n$ and $m(i+1)=$ $[m(i)+1] \times n, \forall n>1$. Therefore, $m(i)$ is on the order of $n^{i}$. Due to the progressive nature of the source code, each packet needs to be decoded successfully before the decoder can start working on subsequent packets, so the number of retransmissions during each failure cannot be very large. In this case, the characteristics of selective repeat strategy clearly do not fit our purposes. Therefore, we use the stop-and-wait scheme in our proposed system and evaluate the retransmission delay accordingly.

If a channel realization incurs no unrecoverable packet error, there will be no repeat requests and the overall delay is identical to that of a system without feedback. Since the existence of feedback allows higher channel code rates, the encoding/decoding is actually faster than in a system without feedback, even in the presence of occasional retransmission.

To verify this comment, we simulated the system specified in Section I-A on a BSC with BER $=0.1$. The optimal code rate for that system is $8 / 19$, at which the block-error rate is $P_{b}=0.0992$. Therefore, the probability of retransmission is approximately 0.1 , and the average number of transmissions for successfully transmitting one channel packet is 1.1.

Although on average it takes 1.1 transmissions to successfully transmit one packet in feedback channels, the transmission time is, in fact, less than that used in systems without feedback. In wireless communication systems, where a mobile station is usually within several miles of a base station, the encoding/decoding time is dominant, so the delay in transmission and propagation can be ignored. In the previous example, the optimal code rate for a ARQ system is $8 / 19$. The average decoding time for one packet is 0.8 of the time for decoding in a system without feedback, whose optimal code rate is $8 / 28$. Considering the average number of transmissions for each packet is 1.1, theoretically, the ARQ system will have smaller average delay than the system without feedback.

Using list decoding [9] in fading channels further complicates the delay analysis, because searching down into the trellis path generates an unpredictable decoding delay. An exact delay analysis in this case is difficult; instead, we present a comparative study based on simulations. Assume we desire a feedback system with average delays comparable to the optimal system without feedback. Because the channel state estimation selects the correct channel code with high probability, and the selected trellis path is often the first one in the list, allowing a maximum of 3-4 retransmissions per failure seems reasonable.

Thus, to maintain an average delay comparable to the scheme without feedback, one can allow a certain number of retransmissions, after which the connection is aborted. To see how restrictive such a termination policy will be, we compute the probability of repeated retransmissions. Simulations are performed in two different channels (see Table III). The typical number of retransmissions is zero, and each failure requires two retransmissions or less with probability better than 0.99 . Therefore, if the number of retransmissions is limited to two or less, the resulting impact on the average end-to-end distortion is negligible.

\section{Numerical Results}

In all experiments, the system specified in Section I-A is used. The source packet length, including CRC and zero padding, is $L^{\prime}=224$. The forward channel is either memoryless or fading, and the feedback channel is assumed error free. The transmission system uses the stop-and-wait feedback scheme.

The first simulation is on a feedback memoryless BSC with $e=0.1$. The optimal channel code rate as a result of (9) is $r=8 / 19$. The PSNR of the received image is listed in Table IV. In the same table, we also list the results of the system given in 
TABLE III

Probability of Retransmission. $e=0.1$, AND the ChannEl DenOted "FC" IS GIVEN UNDER "CHANNEL 1"

\begin{tabular}{|cc|cccc|}
\cline { 3 - 6 } \multicolumn{1}{c|}{} & \multicolumn{4}{|c|}{ Retransmission number } \\
\cline { 3 - 6 } BSC & 0 & 1 & 2 & $\geq 3$ \\
\hline & $0.1 \mathrm{bpp}$ & 0.9070 & 0.0838 & 0.0082 & 0.0010 \\
& $0.5 \mathrm{bpp}$ & 0.9047 & 0.0863 & 0.0082 & 0.0009 \\
& $1.0 \mathrm{bpp}$ & 0.9048 & 0.0860 & 0.0082 & 0.0009 \\
\hline \multirow{3}{*}{ FC } & $0.1 \mathrm{bpp}$ & 0.9029 & 0.0894 & 0.0070 & 0.0007 \\
& $0.5 \mathrm{bpp}$ & 0.9123 & 0.0801 & 0.0069 & 0.0007 \\
& $1.0 \mathrm{bpp}$ & 0.9121 & 0.0804 & 0.0068 & 0.0006 \\
\hline
\end{tabular}

TABLE IV

MEMORYless ChanNel: FeEdBACK Gives UP to ONE DeCiBEl IMPROVEMENT

\begin{tabular}{|c|ccc|}
\cline { 2 - 4 } \multicolumn{1}{c|}{} & \multicolumn{3}{c|}{ Transmission Rate } \\
& 0.1 & 0.5 & 1.0 \\
\hline ARQ & 26.35 & 32.33 & 35.09 \\
FEC & 25.64 & 31.33 & 34.26 \\
\hline
\end{tabular}

TABLE V

PSNR IN FADING CHANNELS WITH AND WITHOUT FEEDBACK

\begin{tabular}{|c|c|c|c|c|}
\hline & \multicolumn{3}{|c|}{ Transmission Rate } \\
\hline & & 0.1 & 0.5 & 1.0 \\
\hline \multirow{2}{*}{ Channel 1} & ARQ & 25.84 & 31.77 & 34.57 \\
\hline & FEC & 25.19 & 30.89 & 33.77 \\
\hline \multirow[t]{2}{*}{ Channel 2} & ARQ & 25.99 & 31.86 & 34.66 \\
\hline & FEC & 25.17 & 30.84 & 33.74 \\
\hline
\end{tabular}

Section II-C. The coding system performance in Table IV confirms our analysis and indicates a $0.7-1.0 \mathrm{~dB}$ PSNR improvement.

The second simulation is performed on two feedback fading channels. The Gilbert-Elliott model is used for the simulation (see Fig. 7). The channel parameters are listed in Table II, where Channel 2 is a slow fading channel compared to Channel 1 . The optimal code rate in this case is $8 / 9$ for the good state and 8/22 for the bad state. The PSNR results are listed in Table V. Again, for comparison, we also list the results of a comparable scheme without feedback [13] in the same table. Results in Table V show about 1-dB PSNR gain. Understandably, there is more gain in the slow fading channel (Channel 2).

\section{DISCUSSION}

This paper concentrates on the transmission of images over noisy channels, but our methodology is not limited to images. The identical approach may be used for progressive transmission of any source over a noisy channel, under an end-to-end distortion criterion.
The success of the proposed algorithms depends critically on a reliable knowledge of channel BERs. It is possible to increase robustness to channel estimation via robust packetization [23], [24], pseudo fixed-length entropy coding [25], or regrouping of the source bits according to sensitivity to errors [26], as well as a number of other approaches. In general, however, robustness comes with a price; sometimes with the loss of progressive transmission, and often with lower performance at the ideal operating point. The desirability of this tradeoff depends largely on the application. Finding suitable measures and tradeoffs for performance versus robustness remains an important open problem in joint source-channel coding.

\section{CONCLUSION}

This paper develops bitrate allocation algorithms for progressive joint source-channel coding of images. Our results are applicable to any progressive source code, and any family of channel codes. It is noteworthy that we do not require rate compatibility of the channel codes. Our parametric approach is constructed using a log-affine empirical model for the decoded block-error rate probability of the channel codes. We used this method to study image transmission in BSCs with and without feedback, under both memoryless and fading conditions.

\section{APPENDIX}

\section{A. Recursive Equation for $P_{i}$}

To solve (3), we need to write $K_{n}$ in terms of $P_{n}$. Using (1), we rewrite the constraint

$$
\prod_{n=1}^{N} P_{n}=P
$$

Therefore, the Lagrangian of (3) is as shown in the equation at the bottom of the page. Taking the derivative of $J$ with respect to $P_{j}$, we have

$$
\begin{aligned}
\frac{\partial J}{\partial P_{j}}= & d_{j-1} \prod_{i=1}^{j-1}\left(1-P_{i}\right) \\
& -\sum_{n=j}^{N-1} d_{n} P_{n+1} \prod_{\substack{i=1 \\
i \neq j}}^{n}\left(1-P_{i}\right)-d_{N} \prod_{\substack{i=1 \\
i \neq j}}^{N}\left(1-P_{i}\right)+\lambda \frac{P}{P_{j}} \\
& j=1,2, \ldots, N .
\end{aligned}
$$

$$
\begin{aligned}
J & =d_{0} P_{1}+\sum_{n=1}^{N-1} d_{n} P_{n+1} \prod_{i=1}^{n}\left(1-P_{i}\right)+d_{N} \prod_{i=1}^{N}\left(1-P_{i}\right)+\lambda\left(\prod_{n=1}^{N} P_{n}-P\right) \\
& =d_{0} P_{1}+\sum_{n=1}^{j-1} d_{n} P_{n+1} \prod_{i=1}^{n}\left(1-P_{i}\right)+\sum_{n=j}^{N-1} d_{n} P_{n+1} \prod_{i=1}^{n}\left(1-P_{i}\right)+d_{N} \prod_{i=1}^{N}\left(1-P_{i}\right)+\lambda\left(\prod_{n=1}^{N} P_{n}-P\right)
\end{aligned}
$$


Setting the derivative to zero yields (12), as shown at the bottom of the page. Dividing both side of $(12)$ by $\left(1-P_{j}\right)$ we will have (13), as shown at the bottom of the page. Similarly, we get (14), as shown at the bottom of the page. Inverting and subtracting (13) from (14), we get

$$
\begin{aligned}
& \left(\frac{1-P_{j+1}}{P_{j+1}}-\frac{1-P_{j}}{P_{j}}\right)(-\lambda P) \\
= & \left(d_{j}\left(1-P_{j+1}\right)-d_{j-1}\right) \prod_{i=1}^{j}\left(1-P_{i}\right)+d_{j} P_{j+1} \prod_{i=1}^{j}\left(1-P_{i}\right) \\
= & \left(d_{j}-d_{j-1}\right) \prod_{i=1}^{j}\left(1-P_{i}\right) .
\end{aligned}
$$

Hence

$$
\left(\frac{1}{P_{j+1}}-\frac{1}{P_{j}}\right)(-\lambda P)=\left(d_{j}-d_{j-1}\right) \prod_{i=1}^{j}\left(1-P_{i}\right)
$$

Notice that when $j=N$, (12) becomes

$$
P_{N}=\frac{-\lambda P}{\left(d_{N-1}-d_{N}\right) \prod_{i=1}^{N-1}\left(1-P_{i}\right)}
$$

which gives $\lambda \leq 0$ because $d_{N-1} \geq d_{N}$ and $0 \leq P, P_{N} \leq 1$. Substituting this back into (15), it easily follows that

$$
P_{j+1} \geq P_{j} \quad \forall j
$$

i.e., the optimal end-to-end block errors are nondecreasing, as one would expect.

\section{B. Mean and Variance of Arrival Process}

Assuming a total of $N$ packets have been transmitted, and denoting the mean and variance of interarrival interval $X$ of two successfully decoded channel packets as $\mu_{x}$ and $\sigma_{x}$, from [20]

$$
\begin{aligned}
\mu & =\frac{N}{\mu_{x}} \\
\sigma & =\sigma_{x} \sqrt{N} \mu_{x}^{-3 / 2} .
\end{aligned}
$$

Notice that

$$
P(X=m)=P_{b}^{m-1}\left(1-P_{b}\right)
$$

then it is easy to compute the mean as

$$
\begin{aligned}
\mu_{x} & =E[X]=\sum_{m=1}^{\infty} m P_{b}^{m-1}\left(1-P_{b}\right) \\
& =\left(1-P_{b}\right) \sum_{m=1}^{\infty} m P_{b}^{m-1} \\
& =\left(1-P_{b}\right) \frac{\partial}{\partial P_{b}}\left(\sum_{m=1}^{\infty} P_{b}^{m}\right) \\
& =\left(1-P_{b}\right) \frac{\partial}{\partial P_{b}}\left(\frac{1}{1-P_{b}}\right) \\
& =\frac{1}{1-P_{b}} .
\end{aligned}
$$

Similarly, we can show that the second moment is

$$
E\left[X^{2}\right]=\sum_{m=1}^{\infty} m^{2} P_{b}^{m-1}\left(1-P_{b}\right)=\frac{1+P_{b}}{\left(1-P_{b}\right)^{2}} .
$$

Hence

$$
\begin{aligned}
\sigma_{x}^{2} & =E\left[X^{2}\right]-(E[X])^{2} \\
& =\frac{1+P_{b}}{\left(1-P_{b}\right)^{2}}-\frac{1}{\left(1-P_{b}\right)^{2}} \\
& =\frac{P_{b}}{\left(1-P_{b}\right)^{2}} .
\end{aligned}
$$

$$
\frac{P_{j+1}}{1-P_{j+1}}=\frac{-\lambda P}{d_{j} \prod_{i=1}^{j+1}\left(1-P_{i}\right)-\sum_{n=j+1}^{N-1} d_{n} P_{n+1} \prod_{i=1}^{n}\left(1-P_{i}\right)-d_{N} \prod_{i=1}^{N}\left(1-P_{i}\right)}
$$


Substituting (18) and (19) into (16) and (17) yields

$$
\begin{aligned}
\mu & =N\left(1-P_{b}\right) \\
\sigma & =\sqrt{P_{b}\left(1-P_{b}\right) N}
\end{aligned}
$$

\section{Maximizing the Effective Transmission Number}

We intend to show that maximizing the effective transmission number is equivalent to maximizing the information bits successfully received. From the previous result, we know that if a total of $N$ packets have been transmitted, the average number of successfully transmitted channel packets is

$$
\mu=\frac{N}{\mu_{x}} \text {. }
$$

Notice that

$$
N=\frac{B}{\frac{L^{\prime}}{r}}
$$

where $L^{\prime}$ is the source packet length (constant) and $r$ is the channel code rate. Therefore

$$
E[M]=\mu=\frac{\frac{B}{L^{\prime}}}{\frac{\mu_{x}}{r}} .
$$

Since $B$ and $L^{\prime}$ are constants

$$
\operatorname{argmax}_{r} E[M]=\operatorname{argmin}_{r} \frac{\mu_{x}}{r} .
$$

We define $\mu_{x} / r$ as the effective transmission number of a successfully transmitted channel packet.

\section{Decoupling the Optimization for Fading Channels With Feedback}

Denote by $k$ the time index of the underlying (hidden) Markov process of the Gilbert-Elliott model, and assume that fading state $S^{(k)} \in \mathcal{S}=\left\{e_{1}, e_{2}, \ldots, e_{I}\right\}$ lasts for a length of $B^{(k)}$ channel bits. In each state $i$, the channel maintains a constant BER $e_{i}$. We assume that the number of bits transmitted in each state $B^{(k)}$ is much longer than the packet length, and ignore the effects of a state change in the middle of a packet. The total number of successfully received packets is $M$. Using a first-order approximation of $D(M)$

$$
\min _{r} E[D] \approx D\left(\max _{r} E[M]\right)
$$

The number of packets successfully received during the $k$ th fading period is denoted $M^{(k)}$. Then

$$
\begin{aligned}
& \min _{r^{(1)}, r^{(2)}, \ldots, r^{(I)}} E[D] \\
\Longleftrightarrow & \max _{r^{(1)}, r^{(2)}, \ldots, r^{(I)}} E[M] \\
= & \max _{r^{(1)}, r^{(2)}, \ldots, r^{(I)}} E\left[M^{(1)}+M^{(2)}+\cdots+M^{(K)}\right] \\
= & \max _{r^{(1)}, r^{(2)}, \ldots, r^{(I)}} E\left[M^{(1)}\right]+E\left[M^{(2)}\right]+\cdots+E\left[M^{(K)}\right] .
\end{aligned}
$$

At the $k$ th fading interval, the fading state is $S^{(k)}=e_{i_{k}}$ with $i_{k} \in \mathcal{I}$. Assuming reliable channel estimation, this state will be known at the receiver. The receiver will then choose a code rate $r^{\left(i_{k}\right)}$. From (22)

$$
E\left[M^{(k)}\right]=\frac{\frac{B^{(k)}}{L^{\prime}}}{\frac{\mu_{x}^{(i)}}{r^{(i)}}}
$$

where $i=i_{k}$ is the state of the channel during fading period $k$, and the expected value is taken with respect to the randomness of the channel, conditioned on the state of the channel.

$$
\begin{aligned}
& \min _{r^{(1)}, r^{(2)}, \ldots, r^{(I)}} E[D] \\
& \Longleftrightarrow \max _{r^{(1)}, r^{(2)}, \ldots, r^{(I)}}\left\{E\left[M^{(1)}\right]+E\left[M^{(2)}\right]+\cdots+E\left[M^{(K)}\right]\right\} \\
& =\max _{r^{(1)}, r^{(2)}, \ldots, r^{(I)}}\left\{\sum_{\substack{k \\
S^{(k)}=e_{1}}} \frac{r^{(1)}}{\mu_{x}^{(1)}} \frac{B^{(k)}}{L^{\prime}}+\sum_{\substack{k \\
S^{(k)}=e_{2}}} \frac{r^{(2)}}{\mu_{x}^{(2)}} \frac{B^{(k)}}{L^{\prime}}\right. \\
& \left.+\cdots+\sum_{\substack{k \\
S^{(k)}=e_{I}}} \frac{r^{(I)}}{\mu_{x}^{(I)}} \frac{B^{(k)}}{L^{\prime}}\right\} \\
& =\max _{r^{(1)}, r^{(2)}, \ldots, r^{(I)}}\left\{\frac{r^{(1)}}{\mu_{x}^{(1)}} \sum_{\substack{k \\
S^{(k)}=e_{1}}} \frac{B^{(k)}}{L^{\prime}}+\frac{r^{(2)}}{\mu_{x}^{(2)}} \sum_{\substack{k \\
S^{(k)}=e_{2}}} \frac{B^{(k)}}{L^{\prime}}\right. \\
& \left.+\cdots+\frac{r^{(I)}}{\mu_{x}^{(I)}} \sum_{\substack{k \\
S^{(k)}=e_{I}}} \frac{B^{(k)}}{L^{\prime}}\right\} \\
& \Longleftrightarrow \max _{r^{(1)}}\left(\frac{r^{(1)}}{\mu_{x}^{(1)}} \sum_{S^{(k)}=e_{1}} \frac{B^{(k)}}{L^{\prime}}\right), \ldots, \\
& \max _{r^{(I)}}\left(\frac{r^{(I)}}{\mu_{x}^{(I)}} \sum_{S^{(k)}=e_{I}} \frac{B^{(k)}}{L^{\prime}}\right) \\
& \Longleftrightarrow \max _{r^{(1)}} \frac{r^{(1)}}{\mu_{x}^{(1)}}, \ldots, \max _{r^{(I)}} \frac{r^{(I)}}{\mu_{x}^{(I)}}
\end{aligned}
$$

where in (23) the first summation is over all $k$ such that $S^{(k)}=$ $e_{1}$, and so on. The cost function consists of a number of nonnegative terms, each of which depend only on one optimization variable. This becomes apparent by noting that the expressions $\sum B^{(k)} / L^{\prime}$ depend on channel realization but are not affected by our choice of code rate. As a result, the optimization can be decoupled.

\section{REFERENCES}

[1] P. G. Sherwood and K. Zeger, "Progressive image coding on noisy channels," in Proc. Data Compression Conf., Snowbird, UT, 1997, pp. 72-81.

[2] _ - "Progressive image coding for noisy channels," IEEE Signal Processing Lett., vol. 4, pp. 189-191, July 1997.

[3] A. Said and W. A. Pearlman, "A new, fast and efficient image codec based on set partitioning in hierarchical trees," IEEE Trans. Circuits Syst. Video Technol., vol. 6, pp. 243-250, June 1996. 
[4] J. Hagenauer, "Rate compatible punctured convolutional codes (RCPC) and their applications," IEEE Trans. Commun., vol. 36, pp. 389-400, Apr. 1988.

[5] L. Qian, D. L. Jones, K. Ramchandran, and S. Appadwedula, "A general joint source-channel matching method for wireless video transmission," in Proc. Data Compression Conf., Snowbird, UT, 1999, pp. 414-423.

[6] V. Chande and N. Farvardin, "Progressive transmission of images over memoryless noisy channels," IEEE J. Select. Areas Commun., vol. 18, pp. 850-860, June 2000 .

[7] P. G. Sherwood, X. Tian, and K. Zeger, "Channel code block length and rate optimization for progressive image transmission," in Proc. Wireless Communications and Networking Conf., New Orleans, LA, 1999, pp. 978-982.

[8] S. B. Wicker, Error Control Systems. Englewood Cliffs, NJ: PrenticeHall, 1995

[9] N. Seshadri and C.-E. W. Sundberg, "List Viterbi decoding algorithms with applications," IEEE Trans. Commun., vol. 42, pp. 313-323, Feb.-Apr. 1994

[10] E. N. Gilbert, "Capacity of a burst-noise channel," Bell Syst. Tech. J., vol. 39, pp. 1253-1265, Sept. 1960.

[11] E. O. Elliott, "Estimates of error rates for codes on burst-noise channels," Bell Syst. Tech. J., vol. 42, pp. 1977-1997, Sept. 1963.

[12] H. Wang and N. Moayeri, "Finite-state Markov channel-A useful model for radio communication channels," IEEE Trans. Veh. Technol., vol. 44, pp. 163-171, Feb. 1995.

[13] J. Lu, A. Nosratinia, and B. Aazhang, "Progressive source-channel coding of images over bursty error channels," in Proc. IEEE ICIP, Chicago, IL, Oct. 1998, pp. 127-131.

[14] N. Matoba, Y. Kondo, and T. Tanaka, "Still image transmission using ARQ over Rayleigh fading channels," Electron. Lett., vol. 32, pp. 803-804, Apr. 1996.

[15] J.-C. Guey and A. S. Khayrallah, "A source-channel coding study of ARQ for the transmission of images," in Proc. IEEE ISIT, Ulm, Germany, 1997, p. 448

[16] V. Kafedziski, "Joint source channel coding of images over frequency selective fading channels with feedback using DCT and multicarrier block pulse amplitude modulation," in Proc. Asilomar Conf. Signals, Systems and Computers, 1988, pp. 37-41.

[17] V. Chande, H. Jafarkhani, and N. Farvardin, "Joint source-channel coding of images for channels with feedback," in Proc. Information Theory Workshop, San Diego, CA, Feb. 1998, pp. 50-51.

[18] V. Chande and N. Farvardin, "A dynamic programming approach to constrained feedback hybrid ARQ design," in Proc. IEEE ISIT, Cambridge, MA, 1998, pp. 286-286.

[19] V. Chande, N. Farvardin, and H. Jafarkhani, "Image communication over noisy channels with feedback," in Proc. IEEE ICIP, vol. 2, Kobe, Japan, 1999, pp. 540-544

[20] R. G. Gallager, Discrete Stochastic Process. Boston, MA: Kluwer, 1995.

[21] P. Zador, "Asymptotic quantization error of continuous signals and the quantization dimension," IEEE Trans. Inform. Theory, vol. IT-28, pp. 139-149, Mar. 1982.

[22] K. Zegerand and A. Gersho, "Pseudo-gray coding," IEEE Trans. Commun., vol. 38, pp. 2147-2158, Dec. 1990

[23] J. K. Rogers and P. C. Cosman, "Robust wavelet zerotree image compression with fixed-length packetization," in Proc. Data Compression Conf., Snowbird, UT, 1998, pp. 418-427.

[24] P. C. Cosman, J. K. Rogers, P. G. Sherwood, and K. Zeger, "Combined forward error control and packetized zerotree wavelet encoding for transmission of images over varying channels," IEEE Trans. Image Processing, vol. 9, pp. 982-993, June 2000.

[25] D. W. Redmill and N. G. Kingsbury, "The EREC: An error-resilient technique for coding variable length blocks of data," IEEE Trans. Image Processing, vol. 5, pp. 565-574, Apr. 1996.

[26] A. A. Alatan, M. Zhao, and A. N. Akansu, "Unequal error protection of SPIHT-encoded image bit streams," IEEE J. Select. Areas Commun., vol. 18, pp. 814-818, June 2000.

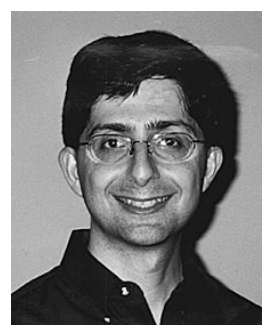

Aria Nosratinia (S'88-M'88) received the B.S. degree from the University of Tehran, Tehran, Iran, in 1988, the M.S. degree from the University of Windsor, Windsor, ON, Canada, in 1991, both in electrical engineering, and the Ph.D. degree in electrical and computer engineering from the University of Illinois at Urbana-Champaign, in 1996.

During the academic year 1995-1996, he was with Princeton University, Princeton, NJ. From 1996 to 1999, he was a Visiting Professor and Faculty Fellow at Rice University, Houston, TX. Since July 1999, he has been on the faculty of the University of Texas at Dallas, where he is currently an Associate Professor of Electrical Engineering. His research interests are in the broad area of communication and information theory, particularly coding and signal processing for the communication of multimedia signals.

Dr. Nosratinia was the recipient of the National Science Foundation Career Award in 2000, and has twice received chapter awards for outstanding service to the IEEE Signal Processing Society. He is currently Associate Editor for IEEE TRANSACTIONS ON IMAGE PROCESSING.

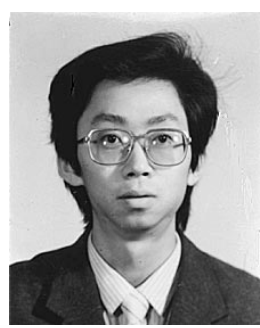

Jin Lu (M'99) received the B.S. and M.S. degrees in control engineering from Tsinghua University, Beijing, China, in 1991 and 1995, respectively, and the $\mathrm{Ph} . \mathrm{D}$. degree in electrical and computer engineering from Rice University, Houston, TX, in 1999.

From 1999 to 2001, he was a Lead DSP Engineer at Improv Systems Inc., Beverly, MA. In 2001, he joined Sony Electronics Inc., San Jose, CA. His research interests include communication theory, information theory, digital signal processing architectures, and rapid prototyping of advanced DSP algorithms.

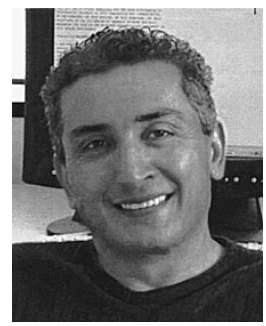

Behnaam Aazhang (S'82-M'82-SM'91-F'99) received the B.S. (with highest honors), M.S., and $\mathrm{Ph} . \mathrm{D}$. degrees in electrical and computer engineering from the University of Illinois at Urbana-Champaign in 1981,1983 , and 1986 , respectively.

From 1981 to 1985, he was a Research Assistant in the Coordinated Science Laboratory, University of Illinois at Urbana-Champaign. In August 1985, he joined the faculty of Rice University, Houston, TX, where he is now the J. S. Abercrombie Professor in the Department of Electrical and Computer Engineering, and the Director of the Center for Multimedia Communications. He has been a Visiting Professor at IBM Federal Systems Company, Houston, TX, the Laboratory for Communication Technology, Swiss Federal Institute of Technology (ETH), Zurich, Switzerland, the Telecommunications Laboratory, University of Oulu, Oulu, Finland, and at the U.S. Air Force Phillips Laboratory, Albuquerque, NM. His research interests are in the areas of communication theory, information theory, and their applications with emphasis on multiple access communications, cellular mobile radio communications, and optical communication networks.

Dr. Aazhang is a member of Tau Beta Pi and Eta Kappa Nu, and is a recipient of the Alcoa Foundation Award in 1993, the National Science Foundation Engineering Initiation Award 1987-1989, and the IBM Graduate Fellowship 1984-1985. He is currently serving on the Houston, TX, Mayor's Commission on Cellular Towers. He has served as the Editor for Spread Spectrum Networks of the IEEE TRANSACTIONS ON COMMUNICATIONS 1993-1998, as the Treasurer of IEEE Information Theory Society 1995-1998, the Technical Area Chair of the 1997 Asilomar Conference, Monterey, CA, the Secretary of the Information Theory Society 1990-1993, the Publications Chairman of the 1993 IEEE International Symposium on Information Theory, San Antonio, TX, and as the Co-Chair of the Technical Program Committee of the 2001 Multidimensional and Mobile Communication (MDMC) Conference, Pori, Finland. 